\title{
Scattering defect in large diameter titaniumdoped sapphire crystals grown by the Kyropoulos technique
}

\author{
Guillaume Alombert-Goget ${ }^{1}$, Yannick Guyot ${ }^{1}$, Abdeldjelil Nehari ${ }^{1}$, \\ Omar Benamara ${ }^{1}$, Nicholas Blanchard ${ }^{1}$, Alain Brenier ${ }^{1}$, \\ Nicolas Barthalay ${ }^{2}$ and Kheirreddine Lebbou ${ }^{1}$ \\ ${ }^{1}$ Univ Lyon, Université Claude Bernard Lyon 1, CNRS, Institut Lumière Matière, F-69622, \\ Villeurbanne, France \\ ${ }^{2}$ Le Rubis SA, BP 16, 38560 Jarrie, Grenoble, France, \\ *Corresponding author: guillaume.alombert-goget@univ-lyon1.fr
}

\begin{abstract}
:
The Kyropoulos technique allows growing large diameter Ti doped sapphire for Chirped pulse amplification laser. A scattering defect peculiar to Kyropoulos grown crystals is presented. This defect is characterized by different techniques: luminescence, absorption measurement, X-ray rocking curve. The impact of this defect to the potential application in chirped pulse amplification CPA laser is evaluated. The nature of this defect is discussed. Modified convexity of the interface is proposed to avoid the formation of this defect and increase the quality of the Ti sapphire crystal.
\end{abstract}

KEYWORDS: Ti-doped sapphire; Kyropoulos method, defects, laser emission.

\section{Introduction}

The excellent mechanical, thermal and optical properties of titanium doped sapphire $\left(\mathrm{Ti}^{3+}\right.$ : $\mathrm{Al}_{2} \mathrm{O}_{3}$ ) allow the development of laser systems with very high average and peak powers. Doping sapphire with $\mathrm{Ti}^{3+}$, confers it broad emission band to allow tunable laser of 
wavelength about $660-1180 \mathrm{~nm}$, and therefore produce laser of short pulses which are less than $10 \mathrm{fs}$ [1][2]. This amplifier material is currently the most widely used in the PetaWatt fs laser chains based Chirped pulse amplification(CPA) technique [3]. The development of these high-power laser systems opens the way to applications based on table-top relativistic laserelectron accelerators [4] or on laser-driven ion sources [5].

New academic projects aimed to obtain powers of the order of tens and hundred Petawatt are under development. Such objectives necessarily require crystals with large diameter size, ultra-high quality, almost without defects, with relatively high concentrations of dopants.

It is therefore significant to develop titanium-doped sapphire with a diameter greater than 100 $\mathrm{mm}$. These crystals must have a good resistance to damage, a good homogeneity of the $\mathrm{Ti}^{3+}$ ions distribution controlled longitudinally and transversely in all the parts. This development requires the control and the well understanding of the various phenomena that occurred during crystallization.

The largest $\mathrm{Al}_{2} \mathrm{O}_{3}: \mathrm{Ti}^{3+}$ crystals existing today in the world have a diameter of the order of $180 \mathrm{~mm}$ with great difficulties of reproducibility. They are obtained by the growth method called HEM (Heat Exchange Method) [6]. But the other techniques such as Kyropoulos method are promising for obtaining large-diameter sapphire crystals able to meet the specifications [3][7].

The current limitations of $\mathrm{Ti}^{3+}: \mathrm{Al}_{2} \mathrm{O}_{3}$ crystals are caused by several reasons. Firstly, reproducing the same growth behaviors with $\mathrm{Ti}^{3+}$ dopants is difficult, the dopants usually show a strong longitudinal and transverse segregation, which degrades the optical quality of the crystal for laser applications [3][8]. Then the segregation results in the propagation of bubbles [9][10], and finally will lead to the thermal stresses which cause great optical damage. 
The defects of single crystals obtained by different drawing methods depend on the growth conditions. Indeed, temperature gradients, convections and surface tensions have much effect on crystal growth. The sapphire melts at $2050{ }^{\circ} \mathrm{C}$, despite its congruent behavior (no change in composition or phase transition during solidification), it is difficult to grow crystals in a stationary state of drawing. The sapphire has a very particular defect: microbubbles of gas in the form of a diameter varying from one micrometer to a few tens of micrometers $[9][10][11][12][13]$. Microbubbles degrade optical properties by decreasing transparency $[9][10][11]$. The presence of colored centers $\left(\mathrm{F}, \mathrm{F}^{+}\right)$is also detrimental for laser application $[14][15]$

FOM (figure of merit) is one of the values most commonly used to evaluate the laser potential for titanium-doped sapphires, which is the ratio of its attenuation coefficients at the laser pump wavelength to the wavelength of the laser emission. To get high FOM values, it is essential to minimize the residual absorption at $800 \mathrm{~nm}$ (center of the laser emission wavelength range) which is attributed to the presence of $\mathrm{Ti}^{3+}-\mathrm{Ti}^{4+}$ pairs [16]. Thus, the difficulties in controlling the valence of titanium $\left(\mathrm{Ti}^{3+}, \mathrm{Ti}^{4+}\right)$ constitute the major problem for using titanium-doped sapphire as a laser material [16][17][18][19]. After annealing the crystal under a reducing atmosphere, it is possible to achieve FOM values higher than 200 of on small diameter components $(<1 \mathrm{~cm})$. It is commonly accepted that sapphires used in a laser cavity must have a FOM greater than 100 [20]. However, for sapphires containing a large quantity of titanium $\left( \pm_{532 \mathrm{~nm}}\right.$ close to or greater than $\left.1 \mathrm{~cm}^{-1}\right)$, it is very difficult to achieve FOM values close to or higher than 100 [16]. On the large diameter crystals (>50 mm) obtained by HEM [19] and Czochralski [8] without post-treatment, FOM values of 100 or slightly higher were measured locally but not homogeneously. Compare to the results published in the literature growing by the other techniques, we have shown that the $100 \mathrm{~mm}$ diameter sapphires obtained by the Kyropoulos method have equivalent FOM values [18]. 
In this paper, we observe and discuss an optical defect responsible for light scattering in titanium-doped sapphires and peculiar to Kyropoulos grown crystal. There are very few references in the literature about this defect. This paper presents its specificity and discuss its the possible nature.

\section{EXPERIMENTAL PROCEDURES}

\section{II.1. Sample preparation}

Ti-doped sapphire single crystals have been grown by the Kyropoulos [7]. The Ti: sapphire crystals used in this study were both grown along a-axis [1120] and m-axis [1100] in argon atmosphere. All the starting titanium concentration in raw material is 0.10 atom $\%$. The details of the growth process have been published in a previous paper [7]. Fig.1 shows the grown crystals obtained by Kyropoulos.

\section{II.3. X-ray rocking curve measurements}

The equipment used is a SmartLab X-ray diffractometer from the Japanese company RIGAKU. It is equipped with a rotating anode of copper, used at $9 \mathrm{~kW}(45 \mathrm{kV}, 200 \mathrm{~mA})$. The optics used, before sample, are a 1D parabolic mirror, a Germanium oriented (2 2 2 0 ) monochromator with 4 reflections, a $5 \mathrm{~mm}$ mask and a $1 \mathrm{~mm}$ slit. The optics used, after sample, are two $1 \mathrm{~mm}$ x $10 \mathrm{~mm}$ slits, $2,5^{\circ}$ Sollers slits, possibly a Germanium oriented (2, 2, 0 ) analyzer with 2 reflections and a scintillation detector. The sample support plate is installed on a double tilts table at $90^{\circ}$ RxRy. A motor placed under the RxRy table, allows the sample to be rotated. With the double tilts table placed above, the axis coincides with the normal to the planes of type $(1,1,0)$.

\section{II.4. transmittance measurements}


The absorption coefficient profiles were obtained after transmittance measurements by the propagation of laser diode emissions at $532 \mathrm{~nm}$ and $808 \mathrm{~nm}$ through the sample. The laser beam diameter on the surface of the sample is about $1 \mathrm{~mm}$. The polarization of the laser beam was linear and setting parallel to the c-axis of the crystal (À polarization) by a Glan-Taylor polarizer. Sample positioning (horizontal and vertical) was performed by linear translation stages with micrometric precision.

\section{II.5. Laser measurements}

The laser cavity was constituted with a plan dichroic input mirror highly transparent near 532 $\mathrm{nm}$ and highly reflective at $800 \mathrm{~nm}$. The output mirror had $20 \%$ transmission near $800 \mathrm{~nm}$. The cavity length was $46 \mathrm{~mm}$. The pump beam from the second harmonic of Quantel Brillant Eazy Nd-YAG laser, at $532 \mathrm{nn}$ wavelength, was focused with a $30 \mathrm{~cm}$ focal length at $1 \mathrm{~cm}$ before the sample. Over than $95 \%$ of the pump power was absorbed by the sample; the remaining pump beam was eliminated after the output mirror with a $750 \mathrm{~nm}$ long pass filter.

\section{II.6. luminescence measurements}

The measurements were performed using an EQ-99 broadband Laser-Driven Light Source (LDLS) from Energetic coupled with a Jobin Yvon Gemini 180 monochromator as excitation source. The luminescence is collected with a UV fiber to a Jobin YvonTriax 320 monochromator and detected by a Jobin Yvon 3000 V CCD camera. This setup allows obtaining excitation emission amplitude map of the sample luminescence.

\section{Results}

\section{III.1. Light scattering}

Large diameter titanium sapphire grown by Kyropoulos technique presents a current defect: a significant scattering area. This scattering area starts from some $\mathrm{cm}$ under the seed and passes 
through almost all the crystal, just stops at the end of the crystal (see figure 1.b and figure 1.c). Observed by the cross section of the crystal, this scattering area is extended on c-plan, it crosses the section by its diameter and has a width of a few mm (figure 1.d). For the crystal grown along a-axis, the scattering area crosses the crystal following a diameter corresponding to m-axis (see figure 1.b). For the crystal grown along $\mathrm{m}$-axis the scattering area staying on cplan (and crosses the crystal following a diameter corresponding to a-axis). This scattering area is not homogeneous, it looks like a cloud in the material with some zones presenting different level of density.

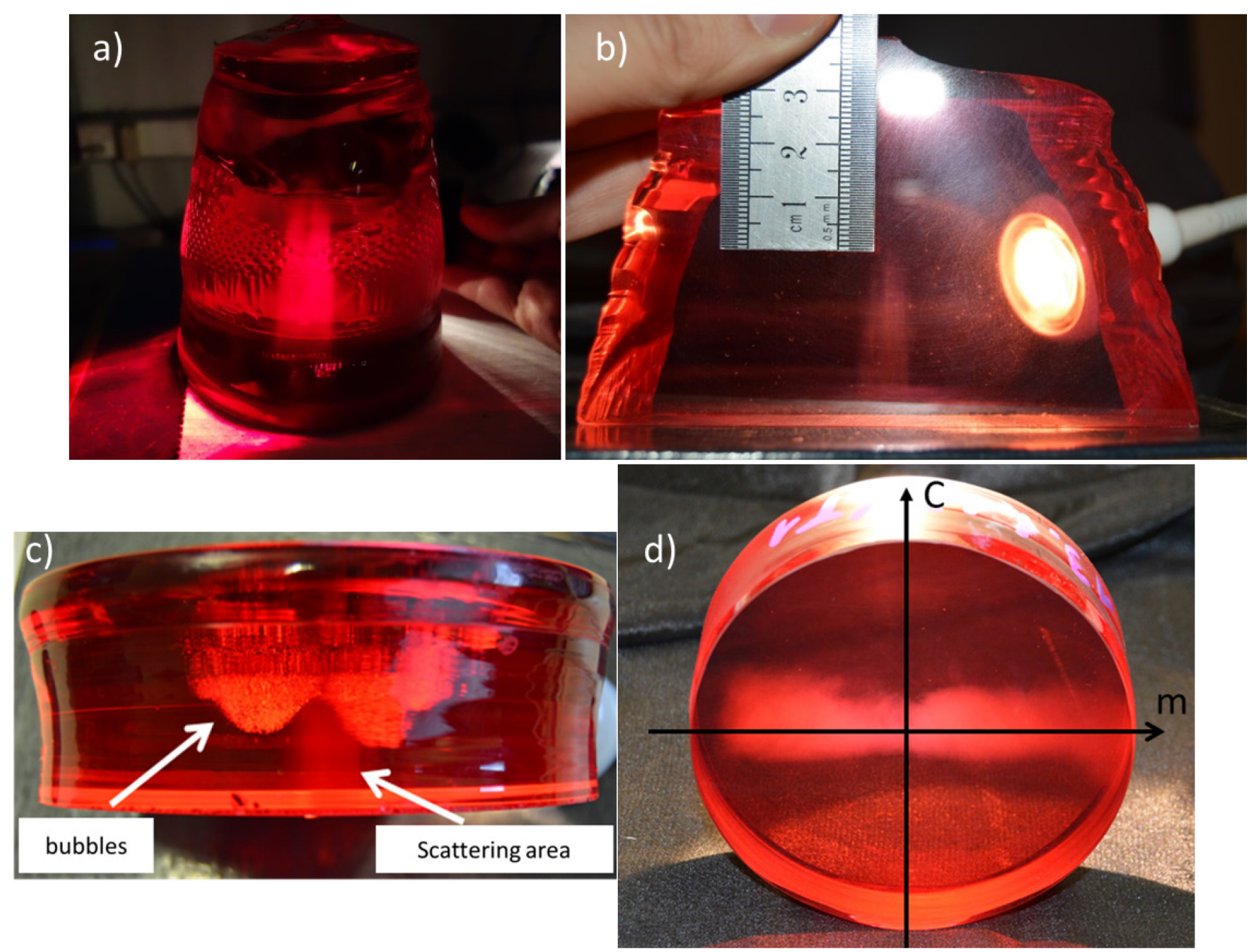

Figure 1: Picture of the kyropoulos sapphire crystal on intense white light. The scattering area is visible in a) the uncut ingot; b) the cutting head part of the ingot; c) the cutting foot part of the ingot, d) cutting central wafer of a crystal grown along a-axis. 
The scattering intensity was depended on the polarization of the incident light and the orientation of the crystal. In figure 4.a, the scattering effect of incident light polarized parallel to the c-axis (À polarization) of the crystal is stronger than in the case of an incident light polarized perpendicular to the c-axis (Â polarization) presented in figure $4 \mathrm{~b}$. Moreover, the scattering is not isotropic and its intensity is different in function of direction of crystallographic axes of the crystal. The scattering is stronger in the direction of the c-axis (see on figure 4c) than in the perpendicular direction (see on figure 4d). This effect of the polarization of the light and the crystallographic aces suggest that the scattering elements in the material are not spherical and present a specific orientation following the crystallographic axes.

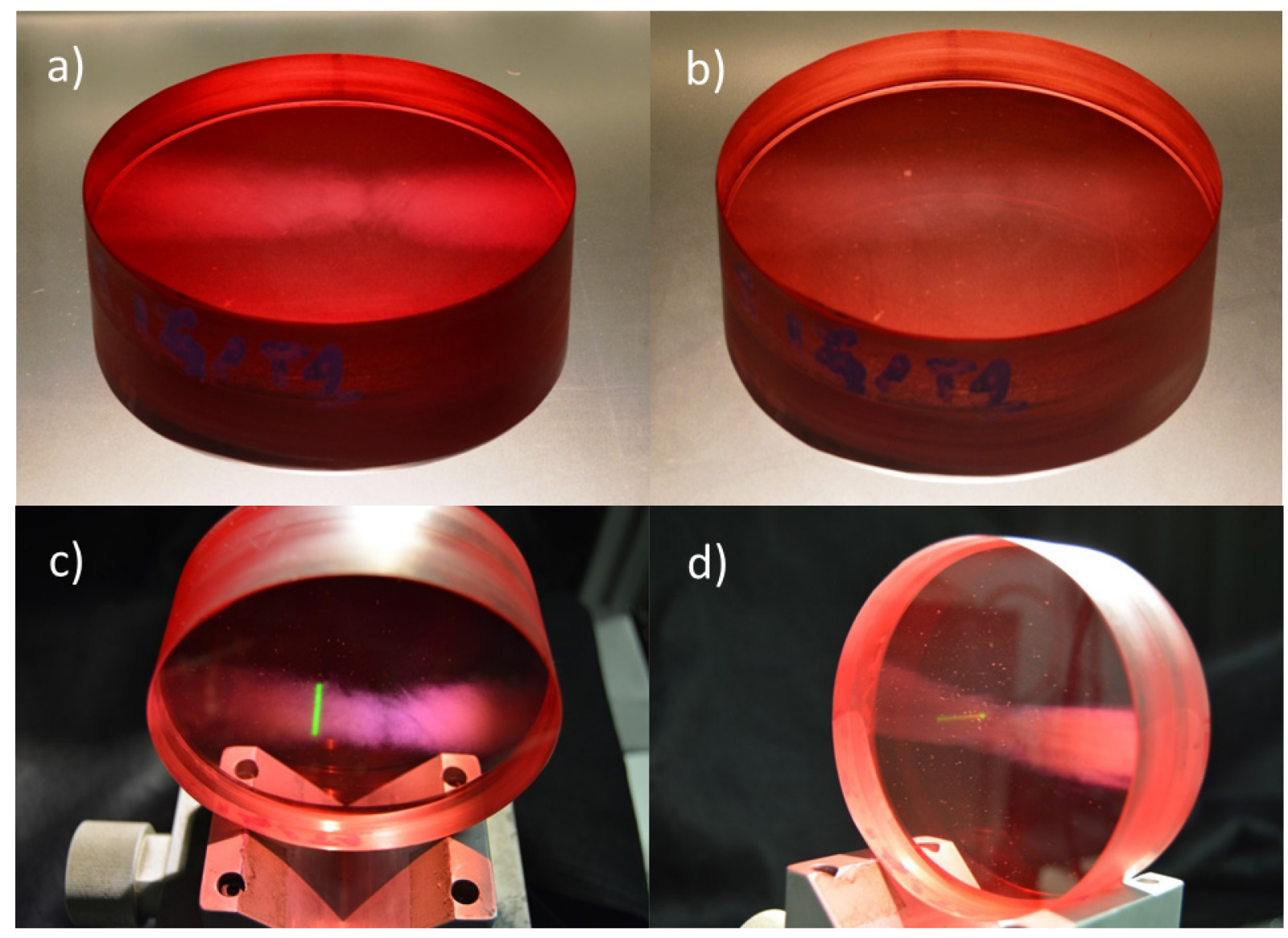


Figure 4: a) Ti-doped sapphire illuminated by light in Àpolarization; b) Ti-doped sapphire illuminated by light in Ãpolarization; c) scattering emission of white light and $532 \mathrm{~nm}$ laser light observed at $45^{\circ}$ to the surface normal in the direction of the $\mathrm{c}$ axis. d) scattering emission of white light and $532 \mathrm{~nm}$ laser light observed at $45^{\circ}$ to the surface normal in the direction perpendicular to c-axis.

The scattering effect was evaluated by transmission measurement at $532 \mathrm{~nm}$ (see on figure 5.a). The transmittance of a laser light in À polarization was measured on two perpendicular chords on the sample (see the schema on figure 5.b). One of them passed through the scattering zone and the other one passed only through free of scattering zone. By comparing the results obtained on both chords, the loss induced by the scattering at $532 \mathrm{~nm}$ is up to $0.1 \mathrm{~cm}^{-1}$

a)

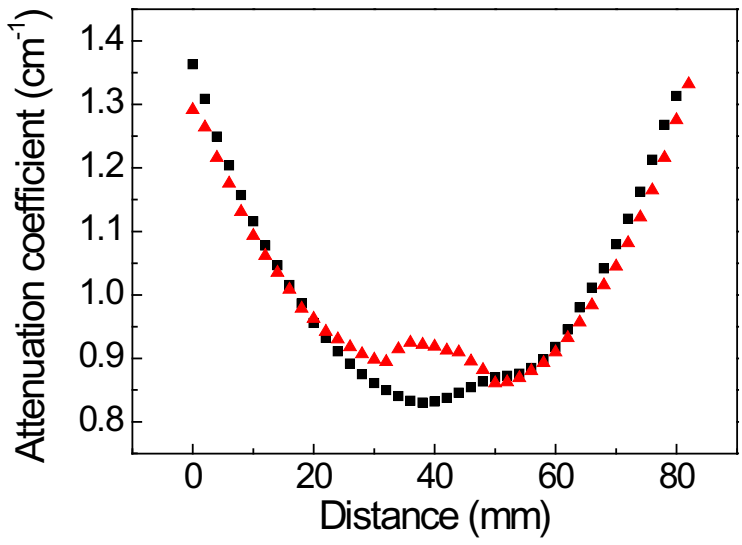

b)

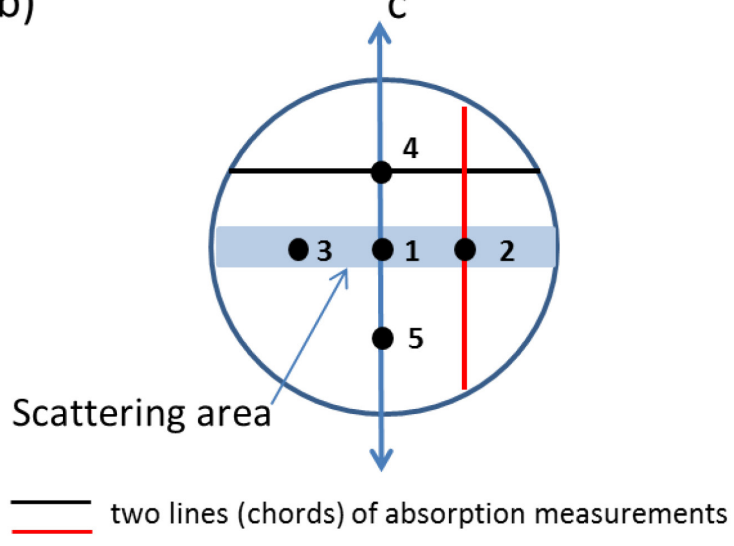

FOM measurements

Figure 5: a) Attenuation coefficient measured in Àpolarization on two chords on the sample at 532 $\mathrm{nm}$ : in black square measurement though a free of scattering zone, in red triangle measurement crossing the scattering zone. b) Schema presenting the two lines (chords) of measurement on the sample in black and red and the different point of FOM measurements.

These losses in transmission measurement have a strong impact on the measured value of the figure of merit. The transmittance at $532 \mathrm{~nm}$ and $808 \mathrm{~nm}$ were measured on five points of the Ti-Sapphire wafer (see the schema in figure 5.b) and reported on table 1. By comparison 
between the points 2 and 3 in the scattering defect and the points 4 and 5 in the free defect area, the losses by scattering corresponding are evaluated. So, if the scattering can induce losses up to $0.1 \mathrm{~cm}^{-1}$ at $532 \mathrm{~nm}$, at $808 \mathrm{~nm}$ the losses achieve $0.025 \mathrm{~cm}^{-1}$. These losses correspond at $532 \mathrm{~nm}$ at $12 \%$ of the absorption of the material but in $808 \mathrm{~nm}$ the losses by scattering is 4 to 5 time more important than the absorption. At $808 \mathrm{~nm}$, the scattering has a very harmful impact on the FOM values. The figure of merit value is defined as:

$$
F O M=\frac{\alpha_{532 \mathrm{~nm}}}{\alpha_{808 \mathrm{~nm}}}
$$

$\pm_{532 \mathrm{~nm}}$ is the attenuation coefficient at $532 \mathrm{~nm}, \pm_{808 \mathrm{~nm}}$ is the attenuation coefficient at $808 \mathrm{~nm}$.

And, the attenuation coefficient is defined as:

$$
\alpha=\alpha_{a}+\alpha_{s}
$$

$\pm_{\mathrm{a}}$ is the absorption coefficient, $\pm_{\mathrm{s}}$ is the scattering coefficient.

At $808 \mathrm{~nm}$ with a scattering coefficient 5 times more important than the absorption coefficient, the value of FOM in scattering area does not depend of the absorption coefficient but depends of the scattering coefficient at $808 \mathrm{~nm}$. Thus in these areas the measured values of the figure of merit are 5 times lower than in other parts of the crystal.

\begin{tabular}{|c|c|c|c|}
\cline { 2 - 4 } \multicolumn{1}{c|}{} & \multicolumn{2}{|c|}{ Attenuation coefficient } & \multirow{2}{*}{ FOM } \\
\cline { 2 - 3 } \multicolumn{1}{c|}{$\left(\mathrm{cm}^{-1}\right)$} & $532 \mathrm{~nm}$ & \\
\hline $\mathbf{1}$ & 0,0209 & 0,7186 & 34 \\
\hline $\mathbf{2}$ & 0,0301 & 0,9010 & 30 \\
\hline $\mathbf{3}$ & 0,0294 & 0,9555 & 32 \\
\hline
\end{tabular}




\begin{tabular}{|c|c|c|c|}
\hline $\mathbf{4}$ & 0,0051 & 0,8575 & 169 \\
\hline $\mathbf{5}$ & 0,0074 & 0,8881 & 120 \\
\hline
\end{tabular}

Table 1: Attenuation coefficients measured at $532 \mathrm{~nm}$ and $808 \mathrm{~nm}$ in Àpolarization on different positions on the sample. The value of the figure of merit (FOM) calculated from the attenuation coefficients are presented for each position of measurements.

\section{III.2. Crystal quality}

The sapphires are perfectly monocrystalline, between two cross polarizer the crystals didn't present any mark of defect (see figure 2) [21] [22]. The scattering area cannot be explained by crystal twinning, grain boundary or any crystalline disorientation. The birefringence proprieties of the crystal are homogenous on all the crystal, so the "inclusion" responsible for the scattering effect must be isotropic.
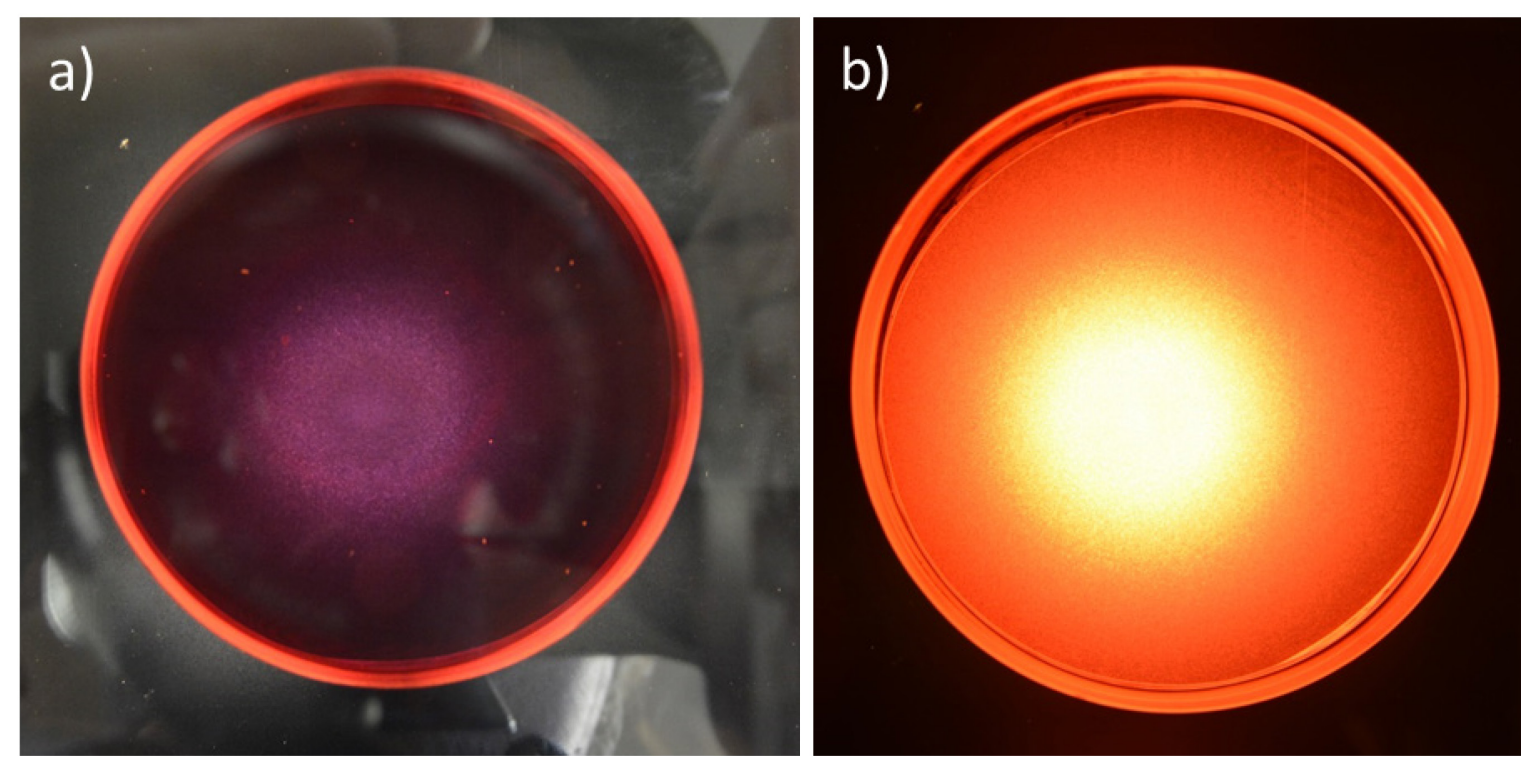

Figure 2: Kyropoulos Ti doped wafer between two cross polarizer. In a) the sapphire is oriented in Off. In b) the sapphire is oriented in On. 
The high crystalline quality of the sapphires grown by Kyropoulos method was confirmed by X-ray diffraction measurement. Figure 3 shows the results of Rocking curve measurements obtained for the plane $(1,1,0)$. The FWHM values for the measurements taken without analyzing are 12.6 arcsec in the area without scattering and $11.9 \operatorname{arcsec}$ in scattering area, respectively. With the analysis FWHM values of the curves for the two areas are 9.7 arcsec. The measured FWHM values are small [23] [24] both in the defect-free zone and in the defective zone. Thus the crystal quality of the sapphire is extremely good in the areas presenting the scattering defects. The quality of the crystal seems even better in areas with a scattering effect. These results are consistent with the measurement of dislocation density realized by Sen et al [25]. They show a lowest level of dislocation density in the central part of the crystal where the scattering defect is present. Due to this high level of crystalline quality, the presence of this scattering area in the crystal can't be explained by a crystallographic defect in the sapphire.
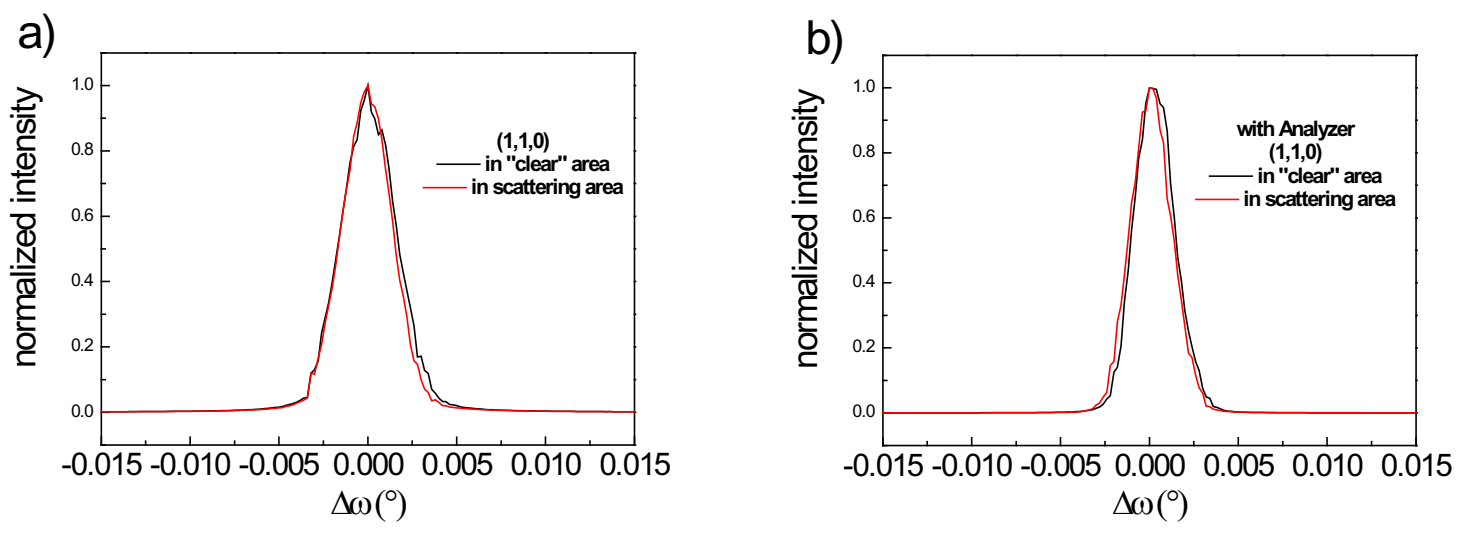

Figure 3: X-ray rocking curve on the plan $(1,1,0)$ in a clear area (without scattering) and in scattering area: measurement without analyzer (a) and with analyzer (b).

\section{III.3. Laser efficiency}


Laser efficiency measurements were realized in order to estimate the possibility to use a sample with this scattering area in laser cavity (see on figure 6). The slope of the laser efficiency on clear area of the sample was estimate at $48.6 \%$. The laser measurement realized on scattering area presented a slope of $38 \%$. The scattering area induces a loss of $22 \%$ of the laser efficiency compared to the free scattering area. The scattering loss induced on the energy of the pump and on the laser emission of the cavity. So a loss of $22 \%$ is not a surprise for an oscillator if you considering the high number of passes inside of the crystal during the laser emission. These measurements confirm that a sapphire containing this scattering defect cannot be used in a laser cavity.

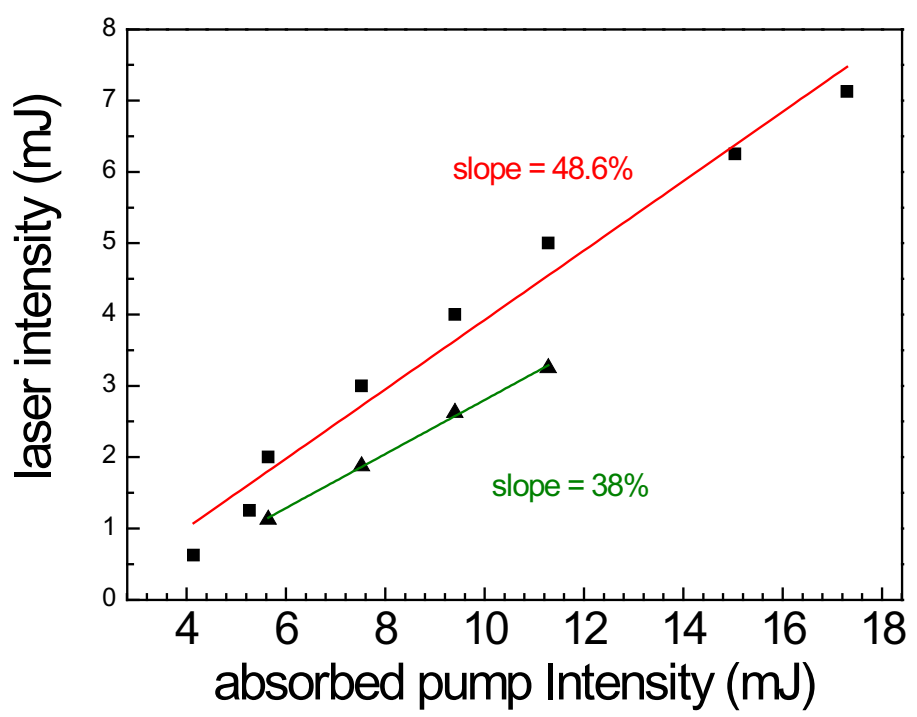

Figure 6: Laser efficiency measurement. The square dots correspond to measurements realized on clear area of the sample. The triangle dots correspond to measurements realized on scattering area of the sample.

As we know, the titanium sapphire crystals of this dimension are not grown to be used in oscillator part but in amplification part of a CPA laser. The losses induced by the scattering area are in energy not so high in absolute value $\left(0.1 \mathrm{~cm}^{-1}\right.$ at $532 \mathrm{~nm}$ and $0.025 \mathrm{~cm}^{-1}$ at $\left.800 \mathrm{~nm}\right)$. With a limited number of pass in the crystal the spurious effect could by less important in 
amplificatory part than in oscillator part. So considering only the losses in energy, the crystal presenting this type of defect could be used in amplification part of a CPA laser. However, the presence of scattering effect in amplification part of a CPA laser can induce a reduction of the temporal contrast of the laser pulse and considering the optical energies involved t may be the source of crystal damage.

\section{III.3. Photoluminescence}

The photoluminescence intensity is not affected by the diffusion of the material. The emission amplitude maps realized on scattering area do not change compare to those realized on the area defect free (see figure 7). They all present the blue emission with the same excitation and emission range. The luminescence properties seem homogenous on the samples. The maps don't show an increase of the $\mathrm{F}$ or $\mathrm{F}^{+}$center emission in particular. If the emission amplitude maps allow a comparison of the spectroscopic properties, it didn't allow a comparison of emission intensity. 
a)

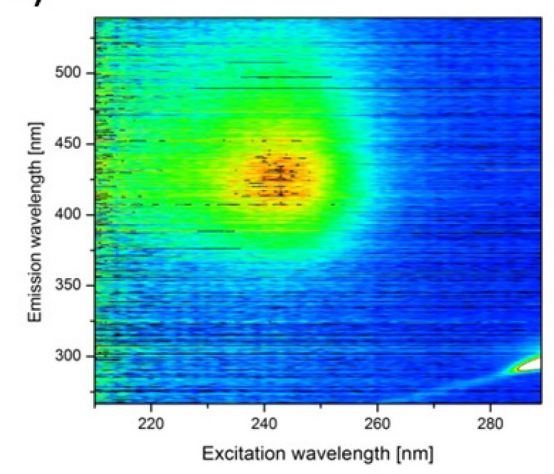

c)

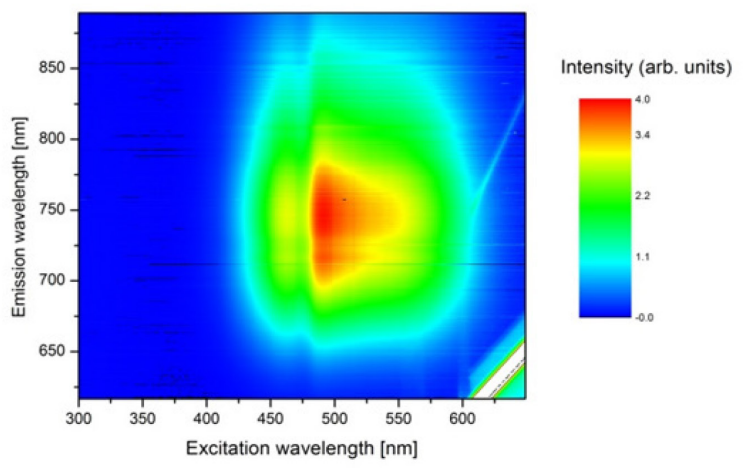

b)
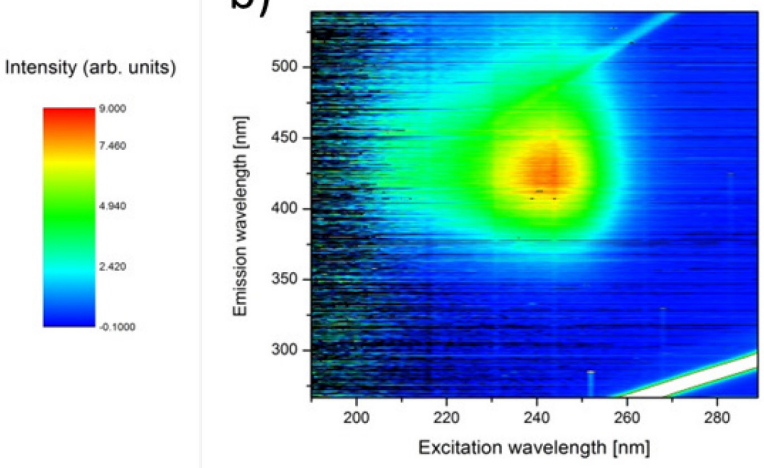

d)

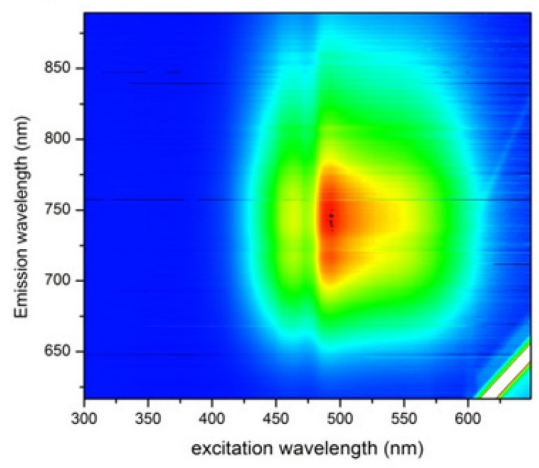

Intensity (arb. units)

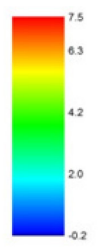

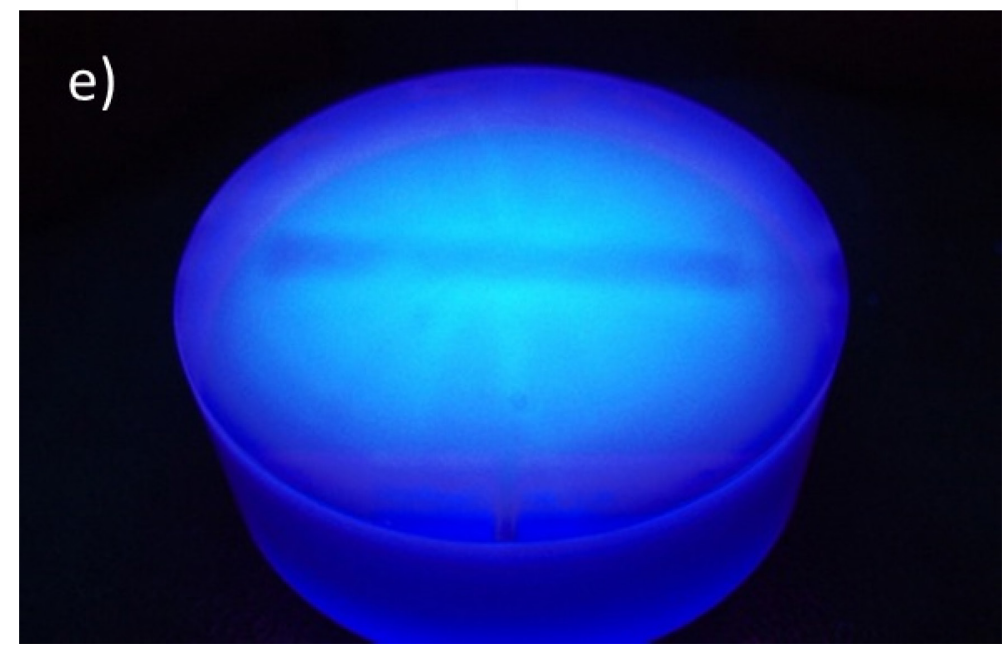

Figure 7: Emission amplitude maps in a) and c) on free defect area, in b) and d) on scattering area. e) Kyropoulos Ti doped wafer on UV lamp at $256 \mathrm{~nm}$.

The blue emission of the Ti-doped sapphire is less intense in the scattering area (see on figure 7e). The defects responsible to this scattering have an impact on the mechanism of the blue emission which is linking with color center and $\mathrm{Ti}^{4+}$ ions [14][15][29][30]. So the amounts of color center or $\mathrm{Ti}^{4+}$ are reducing in the scattering area, which may contradict the explanation 
of an oxygen deficiency in the sapphire. If the stoichiometry of the sapphire is a hypothesis that cannot be dismiss, other possibilities can be evaluated by the comparison between the techniques Kyropoulos and Czochralski as we show below.

\section{Discussion}

There is very few mention of similar defect in the publications. Some scattering centers that look like small bubbles are mentioned by Lacovara et al and attributed to oxygen vacancy [26]. To produce large diameter titanium sapphire crystal the kyropoulos technique used a large diameter molybdenum crucible. In order to avoid the oxidation of the crucible, the furnace integrates an important graphite thermal insulation. The presence of this huge quantity of carbon in the furnace induces a strongly reductive environment [21]. According to Vishnevski- et al [27][28], this reductive environment during growing process induce a oxygen deficiency in the melt stoichiometry, which produces inclusions with an excess of aluminum ions $40-50 \mathrm{~h}$ after the crystallization starting. They evaluated the size of the inclusions at 70-300 $\mathrm{nm}$. Contrary to what they have presented in their publications, we have not been able in the present work to observe these defects by electron microscopy. On the samples extracted from zones having a high scattering density, our measurements did not show the presence of structure which can be attributed to the defects in this range of sizes.

The oxygen deficiency in the stoichiometry of the sapphire should increase the presence of $\mathrm{F}$ center. In absorption spectra, the absorbance attributed to the F center seems increase in the defect area [27]. However the losses by scattering increase the level of the base line in absorption measurement. This is more intense for low wavelength and produces an effect on the F center absorbance band by the increase of its intensity. 
One of the main defects in titanium doped sapphires grown by Czochralski technique are the presence of bubbles as shown in figure 8 already well study previously [10] [13]. But in your knowledge nobody see and report scattering defect in sapphire obtained by Czochralski as presented in this article. They are many differences in the growing process between Czochralski and Kyropoulos. The crystallization takes place in the melt with a highly convex crystallization interface [31]. This interface shape coupled with the segregation effect induces is responsible to a strong gradient of Ti concentration in the crystal [32].
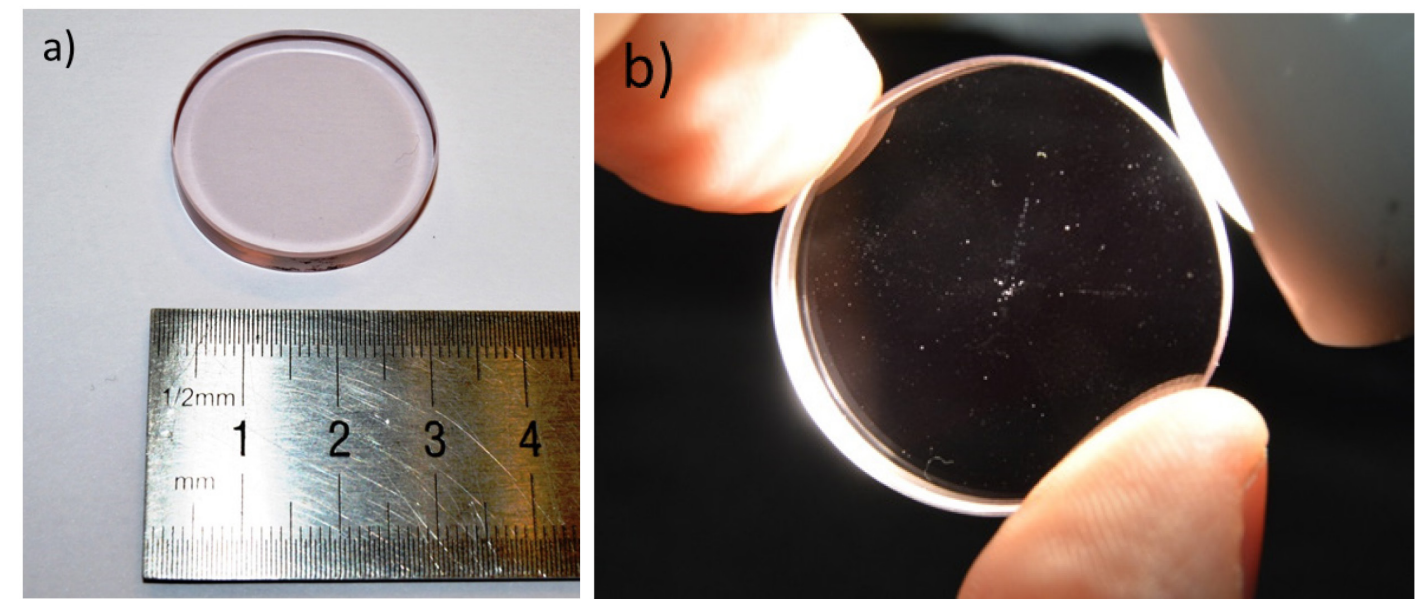

Figure 8: in a titanium sapphire crystal wafer obtains by Czochralski technique; in $\mathrm{b}$ same crystal on strong white light, it exhibited the presence of bubbles but no scattering area like in Kyropoulos wafer.

The interface shape is driving by the thermal gradient in the melt and the crystal due to the isolated configuration and the rotation speed imposed on the crystal. In Kyropoulos technique, the rotation speed currently of $2 \mathrm{rpm}$ is low compared to the rotation speed used in Czochralski technique [10][11]. In Kyropoulos technique, due to this rotation speed and the interface shape, it could be possible than different crystallization speeds in function of the crystal axis occur. Thus, the crystallization in direction of the $\mathrm{c}$ axis is faster than in other direction. This difference of crystallization speeds in function of the crystal axis can explain the creation of defect localized specifically along of on a diameter of the crystal and oriented 
on the c-plan of the crystal. Reduce the convexity of the interface should homogenize the growth rates as a function of the crystallographic axes and potentially eliminate this defect.

Increase the rotation speed at a comparable level to the Czochralski technique can reduce the pressure in the melt below the crystallization interface, and reduce the convexity of the interface. In addition to changing the rotation speed, some other adaptation in the furnace could be considered in order to modify the thermal gradient with the target to reduce the convexity of the interface. Thus, some modifications in the isolation configuration or in the shape of the crucible should be considered to achieve this target.

Obtain an interface more flat would improve the quality of the Ti sapphire for CPA laser application. It allows better radial reparation of the dopant and could reduce the formation of this scattering defect.

\section{Conclusion}

Defects corresponding to the presence of a scattering area in titanium doped sapphire obtained by Kyropoulos technique. This scattering cannot be attributed to the presence of bubbles or induced by a crystallographic defect. In this area a diffusion coefficient of $0.1 \mathrm{~cm}^{-1}$ at $532 \mathrm{~nm}$ and $0.025 \mathrm{~cm}^{-1}$ at $808 \mathrm{~nm}$ are measured. This scattering area has catastrophic effect on the laser properties of the Titanium doped sapphire. The measurements showed that sapphires with this defect could not be used in a cpa laser.

The measures did not allow us to observe or identify the precise nature of the scattering element in the material. The hypothesis of an oxygen deficiency was discussed. But, the spectroscopic measurements, in particular the $\mathrm{F}$ center emissions, do not confirm this explanation. 
By comparing with the Czochralski technique (for which the crystals present no defect), the convex interface shape in the kyropoulos could be responsible of this defect. Thus, increase of the rotation speed in the Kyropoulos, allowing a decrease of the convexity of the interface shape can be a way to eliminate this defect and increase the optical quality of the crystal.

\section{Acknowledgment}

This study was performed in the framework of French national research project FUI TITANSAPHIR with the financial support of the Région Auvergne Rhône-Alpes, project $\mathrm{N}^{\circ}$ 13023257 01. The author would wish to thank the staff at CMTC, Grenoble and in particularly Hervé ROUSSEL for the help with the rocking curve analysis.

\section{References:}

[1] K.F. Wall, A. Sanchez, Titanium sapphire laser, Lincoln Lab. J. 3 (1990) 447-462. doi:10.2184/1sj.21.73.

[2] D.E. Spence, P.N. Kean, W. Sibbett, 60-fsec pulse generation from a self-mode-locked Ti:sapphire laser, Opt. Lett. 16 (1991) 42-44. http://www.ncbi.nlm.nih.gov/pubmed/19773831.

[3] G. Alombert-Goget, K. Lebbou, N. Barthalay, H. Legal, G. Chériaux, Large Ti-doped sapphire bulk crystal for high power laser applications, Opt. Mater. (Amst). 36 (2014) 2004-2006. doi:10.1016/j.optmat.2014.01.011.

[4] E. Esarey, C.B. Schroeder, W.P. Leemans, Physics of laser-driven plasma-based electron accelerators, Rev. Mod. Phys. 81 (2009) 1229-1285. doi:10.1103/RevModPhys.81.1229.

[5] A. Macchi, M. Borghesi, M. Passoni, Ion acceleration by superintense laser-plasma 
interaction, Rev. Mod. Phys. 85 (2013) 751-793. doi:10.1103/RevModPhys.85.751.

[6] D.B. Joyce, F. Schmid, Progress in the growth of large scale Ti:sapphire crystals by the heat exchanger method (HEM) for petawatt class lasers, J. Cryst. Growth. 312 (2010) 1138-1141. doi:10.1016/j.jcrysgro.2009.11.002.

[7] A. Nehari, A. Brenier, G. Panzer, K. Lebbou, J. Godfroy, S. Labor, et al., Ti-Doped Sapphire (Al 2 O 3 ) Single Crystals Grown by the Kyropoulos Technique and Optical Characterizations, Cryst. Growth Des. 11 (2011) 445-448. doi:10.1021/cg101190q.

[8] R. Uecker, D. Klimm, S. Ganschow, P. Reiche, R. Bertram, M. Roßberg, et al., Czochralski growth of Ti:Sapphire laser crystals, in: J.C. Carrano, A. Zukauskas, A.W. Vere, J.G. Grote, F. Kajzar (Eds.), PROC SPIE, 2005: pp. 599006-599006-9. doi:10.1117/12.634322.

[9] E. a Ghezal, H. Li, A. Nehari, G. Alombert-Goget, A. Brenier, K. Lebbou, et al., Effect of Pulling Rate on Bubbles Distribution in Sapphire Crystals Grown by the Micropulling Down ( $\mu-P D)$ Technique, Cryst. Growth Des. 12 (2012) 4098-4103. doi:10.1021/cg300589h.

[10] H. Li, E. a. Ghezal, G. Alombert-Goget, G. Breton, J.M. Ingargiola, A. Brenier, et al., Qualitative and quantitative bubbles defects analysis in undoped and Ti-doped sapphire crystals grown by Czochralski technique, Opt. Mater. (Amst). 37 (2014) 132-138. doi:10.1016/j.optmat.2014.05.012.

[11] H. Li, E. a Ghezal, A. Nehari, G. Alombert-Goget, A. Brenier, K. Lebbou, Bubbles defects distribution in sapphire bulk crystals grown by Czochralski technique, Opt. Mater. (Amst). 35 (2013) 1071-1076. doi:10.1016/j.optmat.2012.12.022.

[12] E.A. Ghezal, A. Nehari, K. Lebbou, T. Duffar, Observation of Gas Bubble 
Incorporation during Micropulling-Down Growth of Sapphire, Cryst. Growth Des. 12 (2012) 5715-5719.

[13] T. Fukuda, Y. Okano, N. Kodama, F. Yamada, S. Hara, D.H. Yoon, Growth of bubblefree Ti-doped A12O3 single crystal by the czochralski method, Cryst. Res. Technol. 30 (1995) 185-188.

[14] G. Alombert-Goget, H. Li, Y. Guyot, A. Brenier, K. Lebbou, Luminescence and coloration of undoped and Ti-doped sapphire crystals grown by Czochralski technique, J. Lumin. 169 (2016) 516-519. doi:10.1016/j.jlumin.2015.02.001.

[15] D. Zhou, C. Xia, Y. Guyot, J. Zhong, X. Xu, S. Feng, et al., Growth and spectroscopic properties of Ti-doped sapphire single-crystal fibers, Opt. Mater. (Amst). 47 (2015) 495-500. doi:10.1016/j.optmat.2015.06.027.

[16] R.L. Aggarwal, A. Sanchez, M.M. Stuppi, R.E. Fahey, A.J. Strauss, W.R. Rapoport, et al., Residual infrared absorption in As-grown and annealed crystals of Ti:A12O3, IEEE J. Quantum Electron. 24 (1988) 1003-1008. doi:10.1109/3.221.

[17] G. Alombert-Goget, F. Trichard, H. Li, C. Pezzani, M. Silvestre, N. Barthalay, et al., Titanium distribution profiles obtained by luminescence and LIBS measurements on Ti: Al2O3 grown by Czochralski and Kyropoulos techniques, Opt. Mater. (Amst). 65 (2017) 28-32. doi:10.1016/j.optmat.2016.09.049.

[18] G. Alombert-Goget, G. Sen, C. Pezzani, N. Barthalay, T. Duffar, K. Lebbou, Large Tidoped sapphire single crystals grown by the kyropoulos technique for petawatt power laser application, Opt. Mater. (Amst). 61 (2016) 21-24. doi:10.1016/j.optmat.2016.04.025.

[19] W.R. Rapoport, C.P. Khattak, Titanium sapphire laser characteristics., Appl. Opt. 27 
(1988) 2677-2684. doi:10.1364/AO.27.002677.

[20] V. Pishchik, L.A. Lytvynov, E.R. Dobrovinskaya, Sapphire, Springer US, Boston, MA, 2009. doi:10.1007/978-0-387-85695-7.

[21] F.J. Bruni, C.-M. Liu, J. Stone-Sundberg, Will Czochralski Growth of Sapphire Once Again Prevail?, Acta Phys. Pol. A. 124 (2013) 213-218. doi:10.12693/APhysPolA.124.213.

[22] F. Farges, G. Panczer, N. Benbalagh, G. Riondet, The Grand Sapphire of Louis XIV and the "Ruspoli" Sapphire: Historical and Gemological Discoveries, Gems Gemol. 51 (2015) 392-409.

[23] A. Jafari, I. Sergueev, D. Bessas, B. Klobes, B.S. Roschin, V.E. Asadchikov, et al., Rocking curve imaging of high quality sapphire crystals in backscattering geometry, J. Appl. Phys. 121 (2017). doi:10.1063/1.4974106.

[24] Y. Wang, S. Liu, G. Peng, S. Zhou, J. Xu, Effects of surface treatment on sapphire substrates, J. Cryst. Growth. 274 (2005) 241-245. doi:10.1016/j.jcrysgro.2004.09.074.

[25] G. Sen, T.N. Tran Caliste, C. Stelian, J. Baruchel, N. Barthalay, T. Duffar, Synchrotron X-ray diffraction imaging studies of dislocations in Kyropoulos grown Ti doped sapphire crystal, J. Cryst. Growth. (2016). doi:10.1016/j.jcrysgro.2016.12.079.

[26] P. Lacovara, L. Esterowitz, M. Kokta, Growth, spectroscopy, and lasing of titaniumdoped sapphire, IEEE J. Quantum Electron. 21 (1985) 1614-1618. doi:10.1109/JQE.1985.1072563.

[27] S.D. Vishnevski-, E. V. Krivonosov, L.A. Litvinov, Specific features of the formation of submicron inclusion in Ti:sapphire, Crystallogr. Reports. 53 (2008) 154-157. 
doi:10.1134/S1063774508010203.

[28] Y.V.K. S.D.Vyshnevskiy, L.A.Lytvynov, Foreign phase inclusions in Ti-sapphire grown in a carbon-containing medium, Funct. Mater. 13 (2006) 238-244.

[29] G. Blasse, J.W.M. Verweij, The luminescence of titanium in sapphire laser material, Mater. Chem. Phys. 26 (1990) 131-137. doi:10.1016/0254-0584(90)90033-7.

[30] V.B. Mikhailik, P.C.F. Di Stefano, S. Henry, H. Kraus, a. Lynch, V. Tsybulskyi, et al., Studies of concentration dependences in the luminescence of Ti-doped Al2O3, J. Appl. Phys. 109 (2011) 53116. doi:10.1063/1.3552943.

[31] C. Stelian, G. Sen, N. Barthalay, T. Duffar, Comparison between numerical modeling and experimental measurements of the interface shape in Kyropoulos growth of Tidoped sapphire crystals, J. Cryst. Growth. 453 (2016) 90-98. doi:10.1016/j.jcrysgro.2016.08.001.

[32] C. Stelian, G. Alombert-Goget, G. Sen, N. Barthalay, K. Lebbou, T. Duffar, Interface effect on titanium distribution during Ti-doped sapphire crystals grown by the Kyropoulos method, Opt. Mater. (Amst). 69 (2017) 73-80. doi:10.1016/j.optmat.2017.04.020. 